\title{
Associations among cardiorespiratory endurance, body mass index and blood pressure in Han Chinese children: results from the 2010 Chinese National Survey On Students' Constitution and Health
}

\author{
Yide Yang ${ }^{1}$, Patrick WC Lau ${ }^{2}$, Jingjing Wang ${ }^{2}$, Bin Dong ${ }^{1,3}$, Lijing Wu ${ }^{1}$, Binh Quach ${ }^{2}$, Del P Wong ${ }^{4}$, \\ Lianguo $\mathrm{Fu}^{5}$, Jun $\mathrm{Ma}^{1}$ and Haijun Wang ${ }^{1}$
}

The associations among cardiorespiratory endurance (CRE), body mass index (BMI) and blood pressure (BP) in children are still unclear. This study aimed to examine the relationships among CRE, BMI and BP in Chinese children. Data were derived from the 2010 Chinese National Survey on Students' Constitution and Health. An endurance run test was used to determine CRE and children were stratified into low and high/moderate CRE groups. BMI was dichotomized into non-overweight and overweight. Among overweight children aged 7-12 years, the risk of high BP (HBP) was significantly higher in the low CRE group than in the high/moderate CRE group (in boys, odds ratio $=1.13,95 \%$ confidence interval: $1.03-1.24, P=0.010$; in girls, odds ratio $=1.18,95 \%$ confidence interval: $1.04-1.33, P=0.010$ ) after adjusting for age, $\mathrm{BMI}$, socioeconomic status and area of residence. However, among all children aged 13-18 years and non-overweight children aged 7-12 years, we did not observe similar results. Higher CRE is associated with lower BP. Overweight children have a significantly higher risk of HBP and low CRE may increase the risk of HBP, independently of BMI, among 7- to 12-year-old overweight children.

Hypertension Research (2016) 39, 799-804; doi:10.1038/hr.2016.63; published online 23 June 2016

Keywords: association; blood pressure; body mass index; cardiorespiratory endurance; children

\section{INTRODUCTION}

There is an increasing prevalence of hypertension worldwide in both adults and children. ${ }^{1,2}$ It has been estimated that the number of people with hypertension will exceed 1.56 billion by $2025 .^{3}$ In China, pediatric blood pressure (BP) and the proportion of children with high BP (HBP) have increased substantially. ${ }^{4,5}$ Elevated BP during childhood and adolescence can carry over into adulthood. ${ }^{6}$ Moreover, persistently HBP or hypertension is a major risk factor for cardiovascular diseases and contributes to $\sim 54 \%$ of stroke cases and $47 \%$ of ischemic heart disease cases. ${ }^{7}$

Although the etiology of elevated BP has not been fully elucidated, high body mass index (BMI) and low cardiorespiratory endurance (CRE) are considered to be important risk factors. ${ }^{8,9}$ CRE has been used as an important predictor of cardiorespiratory health, because it is relatively easy to measure ${ }^{10}$. The associations among CRE, BMI and BP in the adult population have been well documented in cross-sectional studies and longitudinal studies; ${ }^{11,12}$ these studies have revealed that people with either low fitness (CRE) or high fatness (high BMI or high waist circumference) have a higher risk of hypertension. However, this relationship has been found to be inconsistent in pediatric populations. Musa and Williams ${ }^{8}$ have found that both BMI and cardiorespiratory fitness (estimated through the 20 -m shuttle run test) are independent predictors of BP in Nigerian children and, of these two factors, BMI has the more important role. Thomas et al. ${ }^{13}$ have found that the sum of skinfolds is related to BP but that aerobic fitness (estimated by the 20-m shuttle run test) is not related to BP independently of the sum of skinfolds in Welsh children. Similarly, in a US youth study, Kwon et al. ${ }^{14}$ have found that cardiorespiratory fitness (estimated through the submaximal treadmill test) is not associated with BP in boys, and that the effect of cardiorespiratory fitness on cardiovascular risk factors in girls is possibly mediated by BMI. The conflicting results from these limited studies indicate the need for further studies on the associations between CRE, BMI and BP in children and adolescents.

${ }^{1}$ Institute of Child and Adolescent Health, School of Public Health, Peking University Health Science Center, Beijing, China; ${ }^{2}$ Department of Physical Education, Hong Kong Baptist University, Hong Kong, China; ${ }^{3}$ Centre for Chronic Disease, School of Medicine, The University of Queensland, Brisbane, Queensland, Australia; ${ }^{4}$ Sport Science Research Center, Shandong Sport University, Shandong, China and ${ }^{5}$ Department of Preventive Medicine, Bengbu Medical College, Anhui, China

Correspondence: Professor J Ma or Professor H Wang, Institute of Child and Adolescent Health, School of Public Health, Peking University Health Science Center, 38 Xue Yuan Road, Haidian District, Beijing 100191, China.

E-mail: majunt@bjmu.edu.cn or whjun1@bjmu.edu.cn

Received 21 August 2015; revised 29 March 2016; accepted 29 April 2016; published online 23 June 2016 
Therefore, the objective of this study was to examine the effects of $\mathrm{CRE}$ and BMI on BP, to clarify the relationships among CRE, BMI and BP among Chinese children.

\section{MATERIALS AND METHODS \\ Participants}

Data were derived from the 2010 cycle of the Chinese National Survey on Students' Constitution and Health (CNSSCH), a nationally representative cross-sectional survey of students in China that is conducted every 5 years. The participants were selected via stratified multistage sampling among 30 provinces; the selection procedure was the same as that used in the previous $\mathrm{CNSSCH}$ and has been described elsewhere in previous publications. ${ }^{15}$ All participants had a medical examination before recruitment and those with disease or physical/mental deformities were excluded. ${ }^{16}$ The $2010 \mathrm{CNSSCH}$ included 215280 students of Han ethnicity aged 7-18 years. Of these participants, 1538 were excluded because of an absence of BP or endurance run test results. A total of 213742 participants of Han ethnicity were included in the present study.

All subjects were recruited from schools and were stratified by gender, age, area of residence (urban or rural) and socioeconomic status (high, intermediate or low) of the school location, as described previously. ${ }^{15}$

All the procedures in this national survey were in accordance with the principles of the Declaration of Helsinki and the survey was approved by six ministries of China, including the Ministry of Education, the General Administration of Sport, the Ministry of Health, the State Ethnic Affairs Commission, the Ministry of Science and Technology, and the Ministry of Finance. ${ }^{4,16}$ Informed consent was obtained from both the parents and the students. ${ }^{4,16}$

\section{Measurements \\ Anthropometric measurements. Body height $(\mathrm{cm})$ and weight $(\mathrm{kg})$ were measured using metal column height measuring stands and lever scales, respectively. Subjects were required to wear only underwear and to stand up straight on the instruments, while they were barefoot and at ease. BMI was calculated as the weight $(\mathrm{kg})$ divided by the square of the height $\left(\mathrm{m}^{2}\right)$. BMI was classified as non-overweight or overweight (including obese) according to the gender- and age-specific cutoff values for 7- to 18-year-old children developed by the Working Group on Obesity in China ${ }^{17}$ (Supplementary Table S1).}

$B P$ measurements. The method of $\mathrm{BP}$ measurement was described in a previous study. ${ }^{16} \mathrm{BP}$ was measured according to the recommendations of the National High Blood Pressure Education Program Working Group in Children and Adolescents. ${ }^{18}$ An average of three BP measurements during a single visit was calculated for each child. This method has been described elsewhere. ${ }^{4}$

HBP was defined according to the 95th percentile of age- and gender-specific BP in Chinese children ${ }^{19}$ (Supplementary Table S2).

\section{Cardiorespiratory endurance}

CRE was assessed by an endurance run test. Endurance run tests are field tests of fitness and they are one component of the National Fitness Test Battery implemented in China. ${ }^{20}$ The validity of endurance run tests has been examined among young people and the correlation of these results with maximal oxygen consumption $\left(\mathrm{VO}_{2} \mathrm{max}\right)$ is 0.417 and 0.409 for boys $(1000 \mathrm{~m}$ run test) and girls ( $800 \mathrm{~m}$ run test), respectively. ${ }^{21}$

In the present study, an $8 \times 50 \mathrm{~m}$ shuttle run was adopted for children aged $7-12$ years regardless of gender. A 1000- and 800-m run tests were adopted for boys and girls, respectively, aged 13-18 years. The measurements were conducted by trained physical education teachers in accordance with the endurance run test protocol. At least two participants were tested together as a group and they were encouraged to run as quickly as possible. The time to complete the endurance run test was recorded in seconds. The participants who had longer endurance run time were regarded as having lower CRE. CRE was categorized by age- and gender-specific percentiles of endurance run time: low CRE (0-20\%) and high/moderate CRE (20-100\%). A large population cohort study $^{22}$ has shown that subjects in the lowest quintile of cardiorespiratory fitness $(0-20 \%)$ have a significantly higher total mortality and cardiovascular disease mortality than subjects in the other four quintiles (that is, 20-40, 40-60, 60-80 and 80-100\%); thus, we used this health-related cutoff approach for CRE.

\section{Statistical analysis}

For descriptive statistics, we used means \pm s.d. for numerical variables and percentages for categorical variables. Student's $t$-tests were conducted to compare differences in numerical variables between genders and differences in BP across CRE or BMI groups. Pearson's $\chi^{2}$-tests were performed for comparisons of categorical variables. To assess the independent effects of CRE and BMI on BP, participants were further categorized into four BMI-CRE groups: non-overweight and high/moderate CRE, non-overweight and low CRE, overweight and high/moderate CRE, and overweight and low CRE. The differences among the four BMI-CRE groups were assessed via one-way analysis of variance followed by Bonferroni post-hoc analyses. A logistic regression model stratified by weight status and adjusted for age, BMI, area of residence and socioeconomic status was performed to compare the risk of HBP among different CRE groups in both boys and girls. A two-sided $P$-value $<0.05$ was considered to indicate statistical significance.

\section{RESULTS}

Anthropometric characteristics, area of residence, socioeconomic status, BP and HBP prevalence in children of Han ethnicity are shown in Table 1. The overall prevalence of HBP in Chinese children aged $7-12$ years was $11.4 \%$ ( 11.7 in boys and $11.1 \%$ in girls), and that in children aged $13-18$ years was $15.5 \%$ (18.3 in boys and $12.8 \%$ in girls). The prevalence of HBP was significantly higher in the older age group (13-18 years) than in the younger age group (7-12 years). The associations of CRE with BMI and other risk factors of HBP, including area of residence, socioeconomic status and age, are presented in Supplementary Table S3.

\section{Associations of CRE and BMI with BP}

Participants with high/moderate CRE had significantly lower BP than their peers with low CRE $(P<0.001)$, as shown in Table 2 . In boys aged 7-12 years, diastolic BP (DBP) in low CRE participants was $0.9 \mathrm{~mm} \mathrm{Hg}$ higher than that of high/moderate CRE participants $(P<0.001)$ and systolic BP $(\mathrm{SBP})$ in the low CRE group was $0.7 \mathrm{~mm} \mathrm{Hg}$ higher than that in the high/moderate CRE group. DBP and SBP in girls aged 7-12 years were 0.7 and $0.4 \mathrm{~mm} \mathrm{Hg}$ higher, respectively, in the low CRE participants compared with the high/moderate CRE participants $(P<0.001)$. The corresponding increases in DBP and SBP were 0.7 and $0.3 \mathrm{~mm} \mathrm{Hg}$, respectively, in boys aged 13-18 years, and were 0.4 and $0.2 \mathrm{~mm} \mathrm{Hg}$, respectively, in girls aged $13-18$ years (all $P<0.001$ ).

Differences in BP were compared between the two BMI groups of overweight and non-overweight participants (Table 3). In boys aged 7-12 years, DBP and SBP were 4.1 and $7.3 \mathrm{~mm} \mathrm{Hg}$ higher, respectively, in the overweight group than in the non-overweight group. In girls aged 7-12 years, the increases in DBP and SBP in the overweight group compared with the non-overweight group were 3.5 and $6.1 \mathrm{~mm} \mathrm{Hg}$, respectively. These respective values were 3.0 and $7.7 \mathrm{~mm} \mathrm{Hg}$ for boys aged 13-18 years, and 3.3 and $6.5 \mathrm{~mm} \mathrm{Hg}$ for girls aged $13-18$ years (all $P<0.05$ ).

\section{Combined association of CRE and BMI with BP}

To test the combined association of BMI and CRE with BP, a comparison among four BMI-CRE groups was conducted and the results are shown in Table 4. There was an overall significant difference among the four groups. Bonferroni post-hoc analysis for multiple comparisons revealed that in the overweight individuals, the high/moderate CRE group had significantly lower SBP and DBP than 
Table 1 General characteristics of the participants

\begin{tabular}{|c|c|c|c|c|c|c|c|c|}
\hline \multirow[b]{2}{*}{ Variable } & \multicolumn{4}{|c|}{ Aged $7-12$ years $(n=106967)$ mean \pm s.d. } & \multicolumn{4}{|c|}{ Aged $13-18$ years $(n=106775)$ mean \pm s.d. } \\
\hline & Boys $(n=53516)$ & Girls $(n=53451)$ & $t / \chi^{2}$ & P-value & Boys $(\mathrm{n}=53$ 181) & Girls $(n=53594)$ & $t / x^{2}$ & P-value \\
\hline \multicolumn{9}{|c|}{ Area of residence, $\mathrm{N}(\%)$} \\
\hline Urban & $26729(49.9)$ & $26685(49.9)$ & 0.005 & 0.944 & $26559(49.9)$ & $26741(49.9)$ & 0.022 & 0.882 \\
\hline \multicolumn{9}{|l|}{ SES, N (\%) } \\
\hline High & $17745(33.2)$ & $17722(33.2)$ & 0.013 & 0.994 & $17613(33.1)$ & $17857(33.3)$ & 0.695 & 0.707 \\
\hline Intermediate & $17910(33.5)$ & $17874(33.4)$ & & & $17725(33.3)$ & $17871(33.3)$ & & \\
\hline Low & $17861(33.4)$ & $17855(33.4)$ & & & $17843(33.6)$ & 17866 (33.3) & & \\
\hline BMI (kg m $\left.{ }^{-2}\right)$ & $17.4 \pm 3.2$ & $16.6 \pm 2.7$ & 43.710 & $<0.001$ & $20.1 \pm 3.2$ & $19.9 \pm 2.7$ & 11.410 & $<0.001$ \\
\hline $\mathrm{DBP}(\mathrm{mm} \mathrm{Hg})$ & $62.4 \pm 9.9$ & $62.2 \pm 9.8$ & 4.410 & $<0.001$ & $68.5 \pm 9.6$ & $66.4 \pm 8.8$ & 37.730 & $<0.001$ \\
\hline
\end{tabular}

Abbreviations: BMI, body mass index; DBP, diastolic blood pressure; HBP, high blood pressure; SBP, systolic blood pressure; SES, socioeconomic status.

Table 2 BP change according to different level of CRE $(\mathrm{mm} \mathrm{Hg})$

\begin{tabular}{|c|c|c|c|c|}
\hline \multirow[b]{3}{*}{ CRE levels } & \multicolumn{2}{|c|}{ Aged 7-12 years } & \multicolumn{2}{|c|}{ Aged $13-18$ years } \\
\hline & $D B P$ & $S B P$ & $D B P$ & $S B P$ \\
\hline & Mean \pm s.d. & Mean \pm s.d. & Mean \pm s.d. & Mean \pm s.d. \\
\hline \multicolumn{5}{|l|}{ Boys } \\
\hline High/moderate CRE & $61.9 \pm 9.8$ & $99.9 \pm 11.4$ & $68.1 \pm 9.6$ & $111.7 \pm 11.3$ \\
\hline Low CRE & $62.8 \pm 10.0$ & $100.6 \pm 11.7$ & $68.8 \pm 9.5$ & $112.0 \pm 11.9$ \\
\hline$t$ & -9.790 & -6.685 & -8.426 & -2.783 \\
\hline$P$-value & $<0.001$ & $<0.001$ & $<0.001$ & $<0.001$ \\
\hline \multicolumn{5}{|l|}{ Girls } \\
\hline High/moderate CRE & $61.7 \pm 9.7$ & $98.8 \pm 11.2$ & $66.2 \pm 8.8$ & $105.2 \pm 10.4$ \\
\hline Low CRE & $62.4 \pm 9.8$ & $99.2 \pm 11.4$ & $66.6 \pm 8.7$ & $105.4 \pm 10.7$ \\
\hline$t$ & -8.096 & -3.498 & -4.824 & -2.577 \\
\hline$P$-value & $<0.001$ & $<0.001$ & $<0.001$ & $<0.001$ \\
\hline
\end{tabular}

Abbreviations: BP, blood pressure; CRE, cardiorespiratory endurance; DBP, diastolic blood pressure; SBP, systolic blood pressure.

the low CRE group $(P<0.05)$. In overweight boys aged $7-12$ years, the DBP and SBP in the low CRE group were 1.6 and $1.7 \mathrm{~mm} \mathrm{Hg}$ higher, respectively, than those in the high/moderate CRE group $(P<0.05)$. In addition, the corresponding increases in DBP and SBP in girls aged 7-12 years were 1.6 and $1.5 \mathrm{~mm} \mathrm{Hg}$, respectively $(P<0.05)$. In overweight boys aged 13-18 years, the increases in DBP and SBP were 1.1 and $1.2 \mathrm{~mm} \mathrm{Hg}$, respectively, in the low CRE group compared with the high/moderate CRE group $(P<0.05)$. In addition, the corresponding increases in DBP and SBP in girls aged 13-18 years were 1.2 and $2.1 \mathrm{~mm} \mathrm{Hg}$, respectively $(P<0.05)$. In non-overweight individuals, the significant differences in DBP and SBP between the high/moderate and low CRE groups were not consistent. In 7- to 12 -year-old and 13- to 18-year-old non-overweight girls, the DBP values in the low CRE group were 0.6 and $0.3 \mathrm{~mm} \mathrm{Hg}$ higher than those in the high/moderate CRE peers $(P<0.05)$, respectively. The magnitude of the differences in SBP and DBP between high/moderate CRE individuals and low CRE individuals was substantially greater in the overweight group than in the non-overweight group.
Table 3 Comparison of BP between overweight and non-overweight children $(\mathrm{mm} \mathrm{Hg})$

\begin{tabular}{|c|c|c|c|c|}
\hline \multirow[b]{3}{*}{ Nutritional status } & \multicolumn{2}{|c|}{ Aged $7-12$ years } & \multicolumn{2}{|c|}{ Aged $13-18$ years } \\
\hline & $D B P$ & $S B P$ & $D B P$ & $S B P$ \\
\hline & Mean \pm s.d. & Mean \pm s.d. & Mean \pm s.d. & Mean \pm s.d. \\
\hline \multicolumn{5}{|l|}{ Boys } \\
\hline Non-overweight & $61.5 \pm 9.7$ & $98.7 \pm 10.9$ & $68.1 \pm 9.5$ & $110.8 \pm 11.3$ \\
\hline Overweight & $65.6 \pm 10.2$ & $106.0 \pm 12.1$ & $71.1 \pm 9.6$ & $118.5 \pm 11.8$ \\
\hline$t$ & 38.55 & 59.14 & 25.89 & 55.29 \\
\hline$P$-value & $<0.001$ & $<0.001$ & $<0.001$ & $<0.001$ \\
\hline \multicolumn{5}{|l|}{ Girls } \\
\hline Non-overweight & $61.7 \pm 9.6$ & $98.3 \pm 11.0$ & $66.1 \pm 8.7$ & $104.7 \pm 10.4$ \\
\hline Overweight & $65.2 \pm 10.2$ & $104.4 \pm 12.4$ & $69.4 \pm 9.1$ & $111.2 \pm 11.1$ \\
\hline$t$ & 26.23 & 38.17 & 25.04 & 38.88 \\
\hline$P$-value & $<0.001$ & $<0.001$ & $<0.001$ & $<0.001$ \\
\hline
\end{tabular}

Abbreviations: BP, blood pressure; DBP, diastolic BP; SBP, systolic BP.

\section{Independent effect of CRE on HBP}

The results comparing the risk of HBP among the four CRE-BMI groups are listed in Table 5. Overall, overweight children had a higher risk of HBP (ranging from 20.4 to 35.9\%) than non-overweight children (ranging from 8.4 to $16.0 \%$ ). To explore the independent effect of CRE on HBP, we excluded the effect of BMI by adjusting for this parameter. Boys aged 7-12 years in the overweight and low CRE group had a significantly higher risk of HBP than their overweight and high/moderate CRE peers (odds ratio $=1.13,95 \%$ confidence interval: $1.03-1.24, P=0.010)$. In non-overweight boys aged 7-12 years, there was no significant difference among the CRE groups. Similarly, in girls aged 7-12 years, the risk of HBP for the overweight and low CRE group was 1.18 (odds ratio $=1.18,95 \%$ confidence interval: $1.04-1.33$, $P=0.010)$ relative to the overweight and high/moderate CRE group. For non-overweight girls aged 7-12 years, there was no difference in HBP risk between the high/moderate CRE and low CRE groups $(P>0.05)$. 
Table 4 Differences in BP among BMI-CRE groups $(\mathrm{mm} \mathrm{Hg})$

\begin{tabular}{|c|c|c|c|c|c|c|c|}
\hline \multirow[b]{2}{*}{ Gender } & \multirow[b]{2}{*}{$B M I-C R E$ group } & \multicolumn{3}{|c|}{ Aged $7-12$ years $(n=106967)$} & \multicolumn{3}{|c|}{ Aged $13-18$ years $(n=106775)$} \\
\hline & & $\mathrm{n}$ & $D B P$ & $S B P$ & $\mathrm{n}$ & $D B P$ & $S B P$ \\
\hline \multirow{3}{*}{ Boys } & Non-overweight and low CRE & 6390 & $61.7 \pm 10.0$ & $98.6 \pm 10.7$ & 7804 & $68.1 \pm 9.5$ & $110.0 \pm 11.4$ \\
\hline & Overweight and high/moderate CRE & 7490 & $65.0 \pm 10.1$ & $105.4 \pm 12.0$ & 4884 & $70.7 \pm 9.7$ & $118.0 \pm 11.6$ \\
\hline & Overweight and low CRE & 4318 & $66.6 \pm 10.2^{a}$ & $107.1 \pm 12.3^{a}$ & 2839 & $71.8 \pm 9.5^{\mathrm{a}}$ & $119.2 \pm 12.0^{\mathrm{a}}$ \\
\hline \multirow{2}{*}{ Girls } & Overweight and high/moderate CRE & 4190 & $64.7 \pm 10.1$ & $103.9 \pm 12.3$ & 3282 & $69.0 \pm 9.0$ & $110.5 \pm 10.9$ \\
\hline & Overweight and low CRE & 2278 & $66.3 \pm 10.2^{\mathrm{a}}$ & $105.4 \pm 12.5^{a}$ & 1593 & $70.2 \pm 9.3^{a}$ & $112.6 \pm 11.5^{\mathrm{a}}$ \\
\hline
\end{tabular}

Abbreiations: BMI, body mass index; BP, blood pressure; CRE, cardiorespiratory endurance; DBP, diastolic BP; SBP, systolic BP.

a Overweight and high/moderate CRE significantly different from overweight and low CRE.

bNon-overweight and high/moderate CRE significantly different from non-overweight and low CRE.

Table 5 Logistic regression results for the association of BMI and CRE with risk of high BPa

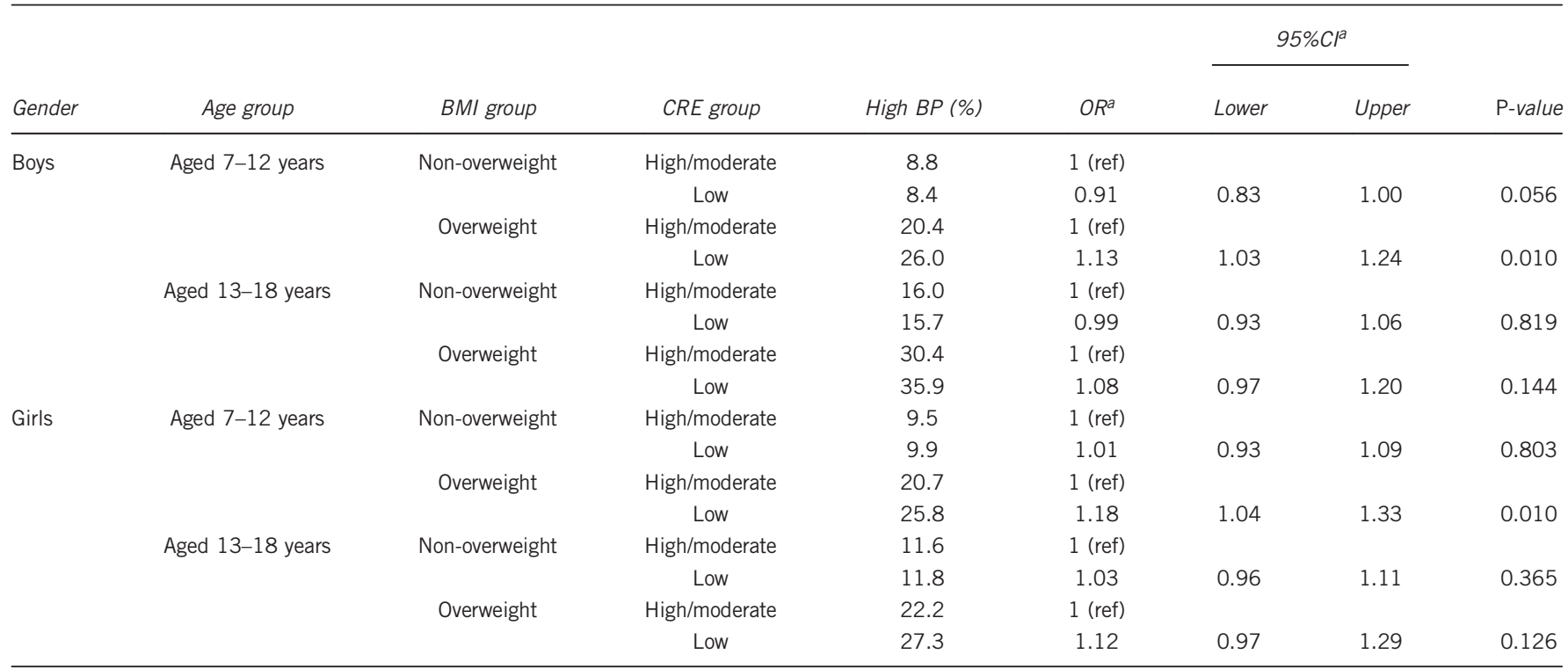

Abbreviations: BMI, body mass index; BP, blood pressure; $\mathrm{Cl}$, confidence interval; $\mathrm{CRE}$, cardiorespiratory endurance; OR, odds ratio; SES, socioeconomic status.

adjusted for age, BMI, SES and area of residence.

In 13- to 18-year-old children, the risk of HBP was not significantly different between the high/moderate CRE group and the low CRE group $(P>0.05)$.

\section{DISCUSSION}

Our study principally showed that in 7- to 12-year-old overweight children, low CRE was associated with an increased risk of HBP independent of BMI. However, in 7- to 12-year-old non-overweight children and 13- to 18-year-old children, no such associations between CRE and HBP were observed.

\section{Prevalence of pediatric HBP}

The overall prevalence of HBP in Chinese children aged 7-12 years and those aged $13-18$ years was $11.4 \%$ and $15.5 \%$, respectively. HBP is becoming a common chronic disease in Chinese children and this condition should warrant increased attention. Substantial evidence has shown that pediatric BP is associated with BP in later life and administering an appropriate intervention in early life is quite important. ${ }^{6}$ It has also been demonstrated in other populations that a higher BMI, that is, overweight or obese status, leads to a higher BP or risk of hypertension. In a Japanese population, overweight and obese subjects have been found to have an approximately twofold greater risk of hypertension than that of normal-weight subjects. ${ }^{23,24}$ Although there is no clear explanation for the mechanism underlying the relationship between overweight/obese status and hypertension, recent research has implied that excessive sympathetic nervous system activity, insulin resistance (insulin/leptin) and inflammation (C-reactive protein) may have an important role. ${ }^{25}$

\section{CRE, BMI and $\mathrm{BP} / \mathrm{HBP}$}

According to our results, irrespective of BMI, subjects with high/moderate CRE had more favorable BP profiles than their low CRE peers across age and gender strata. In addition, being overweight and having low CRE had a combined effect of increasing BP. These findings were consistent with those of similar studies ${ }^{26,27}$ that did not adjust for BMI. The study of Wang et al. ${ }^{26}$ in Wuhan, China, has found that fitness (estimated by the 20-m shuttle run test) and the fatness (waist circumference or BMI) are associated with cardiovascular risk (metabolic risk score) in children. A study by Ogunleye et al. ${ }^{27}$ evaluating 5983 school children aged $10-16$ years 
from eastern England using a $20-\mathrm{m}$ shuttle run test has reported similar results.

However, further analysis has shown that low CRE increases the risk of HBP independently of BMI in overweight children aged 7-12 years. Crump et al. ${ }^{28}$ have performed a cohort study confirming that low physical fitness (aerobic capacity) is associated with a subsequent increased risk of hypertension, even after adjusting for BMI and other potential confounders. However, our study showed that in 13- to 18-year-old children, the effect of low CRE on the risk of HBP was dependent on BMI. After adjusting for BMI, the difference in HBP risk between the low and high/moderate CRE groups was no longer significant. Similar to our results, Kwon et al. ${ }^{14}$ have reported that in adolescents aged 12-19 years involved in NHANES 1999-2002, cardiorespiratory fitness was associated with SBP, but this result was no longer significant after adjusting for BMI.

There are several differences between our study and other similar studies. First, in our study, the CRE was measured by using an endurance run test, which was a common CRE test in the CNSSCH from 1985 to 2010. However, different measures of CRE (for example, the $1.6-\mathrm{km}$ endurance run test, $20-\mathrm{m}$ shuttle run test, submaximal treadmill test or 3-min step test) have been used in other studies. In addition, the discretization of CRE variables (for example, into two categories, quartiles or quintiles, or applying specific cutoff values) was different among studies. In the present study, the discretization of CRE was based on a large population cohort study of the relationship between CRE and cardiovascular risk, which showed that those with low CRE (the lowest quintile, 0-20\%) had an unusually higher risk of mortality (relative risk $=3.44$ and 4.65 for men and women, respectively) and risk of cardiovascular disease, ${ }^{22}$ and those results have been confirmed by another large cohort study. ${ }^{29}$

The mechanism underlying the association between CRE and BP has not been fully elucidated. One possible reason for this association is that the benefits of CRE are mediated by circulating endothelial progenitor cells that are involved in vascular repair and that improve vascular function, ${ }^{30}$ because these processes are significantly improved after exercise training. ${ }^{31,32}$ Another possible reason is that higher CRE is associated with lower arterial stiffness and greater arterial distensibility and compliance, which may influence BP in adults and children. $^{33-35}$ Exercise training can decrease SBP or DBP through neurohormonal adaptions, such as decreases in the levels of renin, angiotensin and plasma norepinephrine, which may be mediated by improved CRE. ${ }^{36}$

As high/moderate CRE is significantly associated with low BP in overweight or obese 7 - to 12-year-old children but not in 13- to 18-year-old children, our results highlighted the importance of exercise intervention in early life to improve the BP profile. Our results also indicated that an exercise intervention may reduce the risk of HBP without changing BMI. Although the exact reason for the adverse effect of obesity on BP in young children and especially among adolescents is unclear, one plausible explanation for this phenomenon might involve the relatively short duration of obesity for young children. However, further study is needed to evaluate this possibility.

Our results suggested that high CRE, independently of BMI, may explain the additional beneficial effect of increased CRE on HBP risk in 7- to 12-year-old overweight children. Physical activity interventions are commonly used in weight management to improve CRE and these interventions are relatively safe and inexpensive compared with anti-hypertension treatment. Notably, weight control is a widely accepted and highly beneficial intervention, but achieving an adequate level of CRE may provide an additional health benefit, in particular in 7- to 12-year-old overweight children.

\section{Strengths and limitations}

Our study was a nationally representative study that consisted of Chinese children and adolescents ranging from 7 to 18 years old, which examined the effects of CRE and BMI on BP to produce generalizable results. Our findings implied that the increasing CRE may improve the BP profiles of overweight children. Thus, our findings may provide a new perspective and direction for future interventions. However, several limitations should also be considered when interpreting our results. This was a cross-sectional study that cannot prove a causal relationship. Therefore, a further longitudinal, prospective study in the pediatric population should be conducted to confirm this relationship. Second, the endurance run test, as an indicator of cardiovascular endurance, is measured differently worldwide, and this discrepancy may affect the comparability of these results with those from other countries. Third, the population of this study is limited to the Han ethnicity, which comprises $90 \%$ of the population in China. The situation of other ethnic groups, which may be different from Han ethnicity, still calls for further exploration.

\section{CONCLUSION}

In summary, overweight children aged 7-12 years had a significantly higher risk of $\mathrm{HBP}$ and low CRE levels increased this risk independently of BMI. Given the distinct effects of CRE in different age groups and weight status groups, specific health promotions and therapeutic interventions aimed at improving fitness and controlling pediatric obesity in a diverse population may contribute to the maximization of public health benefits.

\section{CONFLICT OF INTEREST}

The authors declare no conflict of interest.

\section{ACKNOWLEDGEMENTS}

We acknowledge WK Liao, WH Xing, X Zhang and the members of the CNSSCH for providing access to the survey data. We also appreciate the cooperation of the students who participated in the survey. The data analysis was supported by grants from the Research Special Fund for Public Welfare Industry of Health (201202010), the National Natural Science Foundation of China (81573170) and the National Health and Medical Research Council of Australia (APP1045000).

1 Ibrahim MM, Damasceno A. Hypertension in developing countries. Lancet 2012; 380 611-619.

2 Flynn J. The changing face of pediatric hypertension in the era of the childhood obesity epidemic. Pediatr Nephrol 2013; 28: 1059-1066.

3 Chockalingam A, Campbell NR, Fodor JG. Worldwide epidemic of hypertension. Can J Cardiol 2006; 22: 553-555.

4 Dong B, Wang HJ, Wang Z, Liu JS, Ma J. Trends in blood pressure and body mass index among Chinese children and adolescents from 2005 to 2010. Am J Hypertens 2013; 26: 997-1004.

$5 \mathrm{Xi} \mathrm{B}$, Liang Y, Mi J. Hypertension trends in Chinese children in the national surveys, 1993 to 2009. Int J Cardiol 2013; 165: 577-579.

6 Chen X, Wang Y. Tracking of blood pressure from childhood to adulthood: a systematic review and meta-regression analysis. Circulation 2008; 117: 3171-3180.

7 Lawes CM, Vander HS, Rodgers A. Global burden of blood-pressure-related disease, 2001. Lancet 2008; 371: 1513-1518.

8 Musa DI, Williams CA. Cardiorespiratory fitness, fatness, and blood pressure associations in Nigerian youth. Med Sci Sports Exerc 2012; 44: 1978-1985.

9 Eisenmann JC, Welk GJ, Ihmels M, Dollman J. Fatness, fitness, and cardiovascular disease risk factors in children and adolescents. Med Sci Sports Exerc 2007; 39 . 1251-1256.

10 Committee on Fitness Measures and Health Outcomes in Youth. Fitness Measures and Health Outcomes in Youth. National Academies Press (US): Washington (DC), 2012.

11 Vranian MN, Keenan T, Blaha MJ, Silverman MG, Michos ED, Minder CM, Blumenthal RS, Nasir K, Meneghelo RS, Santos RD. Impact of fitness versus obesity on routinely measured cardiometabolic risk in young, healthy adults. Am J Cardiol 2013; 111: 991-995. 
12 Chen J, Das S, Barlow CE, Grundy S, Lakoski SG. Fitness, fatness, and systolic blood pressure: data from the Cooper Center Longitudinal Study. Am Heart J 2010; 160: $166-170$.

13 Thomas NE, Cooper SM, Williams SP, Baker JS, Davies B. Relationship of fitness, fatness, and coronary-heart-disease risk factors in 12- to 13-year-olds. Pediatr Exerc Sci 2007; 19: 93-101.

14 Kwon S, Burns TL, Janz K. Associations of cardiorespiratory fitness and fatness with cardiovascular risk factors among adolescents: the NHANES 1999-2002. J Phys Act Health 2010; 7: 746-753.

$15 \mathrm{Ji} \mathrm{CY}$, Cheng TO. Prevalence and geographic distribution of childhood obesity in China in 2005. Int J Cardiol 2008; 131: 1-8.

16 Dong B, Ma J, Wang HJ, Wang ZQ. The association of overweight and obesity with blood pressure among Chinese children and adolescents. Biomed Environ Sci 2013; 26: 437-444.

$17 \mathrm{Ji} \mathrm{CY}$. Report on childhood obesity in China (1)-body mass index reference for screening overweight and obesity in Chinese school-age children. Biomed Environ Sci 2005; 18: 390-400.

18 NHBPEP. The fourth report on the diagnosis, evaluation, and treatment of high blood pressure in children and adolescents. Pediatrics 2004; 114: 555-576.

19 Mi J, Wang T, Meng L, Zhu G, Han S, Zhong Y, Liu G, Wan Y, Xiong F, Shi J, Yan W, Zhou P. Development of blood pressure reference standards for Chinese children and adolescents. Chin J Evid Based Pediatr 2010; 5: 4-14.

20 Deng XF, Huang Y, Deng MY, Qu SH. A comparative study of fitness test batteries between school-based physical education programs in the USA and the People's Republic of China. Int Sports Stud 2003; 25: 5-22.

21 Zhai S, Xu C. Validity of cardiorespiratory endurance test index of college students' health related physical fitness program. J Wuhan Inst Phys Educ 2011; 45: 87-90.

22 Blair SN, Kohl HR, Paffenbarger RJ, Clark DG, Cooper KH, Gibbons LW. Physical fitness and all-cause mortality. A prospective study of healthy men and women. JAMA 1989; 262: 2395-2401.

23 Nagai M, Ohkubo T, Murakami Y, Takashima N, Kadota A, Miyagawa N, Saito $Y$, Nishi N, Okuda N, Kiyohara Y, Nakagawa H, Nakamura Y, Fujiyoshi A, Abbott RD, Okamura T, Okayama A, Ueshima H, Miura K. Secular trends of the impact of overweight and obesity on hypertension in Japan, 1980-2010. Hypertens Res 2015; 38: 790-795.

24 Kuwahara E, Asakura K, Nishiwaki Y, Komatsu H, Nakazawa A, Ushiku H, Maejima F, Nishigaki Y, Hasegawa T, Okamura T, Takebayashi T. Steeper increases in body mass index during childhood correlate with blood pressure elevation in adolescence: a longterm follow-up study in a Japanese community. Hypertens Res 2014; 37: 179-184.
25 Fujii M, Ohnishi H, Saitoh S, Akasaka H, Miura T, Mori M. The combination of abdominal obesity and high-sensitivity C-reactive protein predicts new-onset hypertension in the general Japanese population: the Tanno-Sobetsu study. Hypertens Res 2015; 38: 426-432.

26 Wang PG, Gong J, Wang SQ, Talbott EO, Zhang B, He QQ. Relationship of body fat and cardiorespiratory fitness with cardiovascular risk in Chinese children. PLoS ONE 2011; 6: e27896.

27 Ogunleye AA, Sandercock GR, Voss C, Eisenmann JC, Reed K. Prevalence of elevated mean arterial pressure and how fitness moderates its association with BMI in youth. Public Health Nutr 2013; 16: 2046-2054.

28 Crump C, Sundquist J, Winkleby MA, Sundquist K. Interactive effects of physical fitness and body mass index on the risk of hypertension. JAMA Intern Med 2016; 176: 210-216.

29 Grundy SM, Barlow CE, Farrell SW, Vega GL, Haskell WL. Cardiorespiratory fitness and metabolic risk. Am J Cardiol 2012; 109: 988-993.

30 Steiner S, Niessner A, Ziegler S, Richter B, Seidinger D, Pleiner J, Penka M, Wolzt M, Huber K, Wojta J, Minar E, Kopp CW. Endurance training increases the number of endothelial progenitor cells in patients with cardiovascular risk and coronary artery disease. Atherosclerosis 2005; 18: 305-310.

31 Clarkson P, Montgomery HE, Mullen MJ, Donald AE, Powe AJ, Bull T, Jubb M, World M, Deanfield JE. Exercise training enhances endothelial function in young men. J Am Coll Cardiol 1999; 33: 1379-1385.

32 Fernandez JM, Rosado-Alvarez D, Da SGM, Rangel-Zuniga OA, Landaeta-Diaz LL, Caballero-Villarraso J, Lopez-Miranda J, Perez-Jimenez F, Fuentes-Jimenez F. Moderate-to-high-intensity training and a hypocaloric Mediterranean diet enhance endothelial progenitor cells and fitness in subjects with the metabolic syndrome. Clin Sci (Lond) 2012; 123: 361-373.

33 Gando Y, Kawano H, Yamamoto K, Sanada K, Tanimoto M, Oh T, Ohmori Y, Miyatani M, Usui C, Takahashi E, Tabata I, Higuchi M, Miyachi M. Age and cardiorespiratory fitness are associated with arterial stiffening and left ventricular remodelling. J Hum Hypertens 2010; 24: 197-206.

34 Reed KE, Warburton DE, Lewanczuk RZ, Haykowsky MJ, Scott JM, Whitney CL, McGavock JM, McKay HA. Arterial compliance in young children: the role of aerobic fitness. Eur J Cardiovasc Prev Rehabil 2005; 12: 492-497.

35 Huynh QL, Blizzard CL, Raitakari O, Sharman JE, Magnussen CG, Dwyer T, Juonala M, Kahonen M, Venn AJ. Vigorous physical activity and carotid distensibility in young and mid-aged adults. Hypertens Res 2015; 38: 355-360.

36 McAuley PA, Beavers KM. Contribution of cardiorespiratory fitness to the obesity paradox. Prog Cardiovascu Dis 2014; 56: 434-440.

Supplementary Information accompanies the paper on Hypertension Research website (http://www.nature.com/hr) 